\title{
Research on Filling Channel of ESC by Fixed Consumable Electrode Filling Method
}

\author{
Wang Jun ${ }^{1}$, Chang Guowei ${ }^{1}$, Chen Rui ${ }^{2}$ \\ ${ }^{1}$ College of Material Science and Engineering, Liaoning University of Technology, Jinzhou, China \\ ${ }^{2}$ Shenyang Research Institute of Foundry, Shenyang, China
}

\section{Email address:}

cailiaowangjun@sina.com (Wang Jun),cgw@Inut.edu.cn (Chang Guowei), chenrui320@sina.com (Chen Rui)

\section{To cite this article:}

Wang Jun, Chang Guowei, Chen Rui. Research on Filling Channel of ESC by Fixed Consumable Electrode Filling Method. Science Discovery. Vol. 6, No. 4, 2018, pp. 263-267. doi: 10.11648/j.sd.20180604.16

Received: June 17, 2018; Accepted: August 3, 2018; Published: August 10, 2018

\begin{abstract}
This study takes the consumable electrode, slag bath, and ingot as investigated objects. Mathematical models is established based on the convection thermal transfer equation of the slag bath, the thermal conduction equation of the ingot, and the melt flowing equation,meanwhile the appropriate electromagnetic field, thermal boundary conditions are applied, The numerical simulation study on influence of process parameters on filling channels between metal pools in electroslag casting process with fixed consumable electrode filling method are performed by using large finite element analysis software ANSYS. Size of casting ingot is $400 \times 70 \times 400$, the research show that when the current density of mobile consumable electrode is greater than or equal to $0.5 \mathrm{~A} / \mathrm{mm}^{2}$, the electrode spacing is less than or equal to $0.15 \mathrm{~m}$, and the electrode thickness is greater than or equal to $0.035 \mathrm{~m}$, the two metal pools are connected, molten metal of moved consumable electrode casting successfully supply the metal pool of fixed consumable electrode through filling channel, make sure that casting is forming under stable electroslag conditions. the minimum sectional area of filling channel is increased with the increase of current density of the mobile consumable electrode and the cross-sectional area of the consumable electrode.
\end{abstract}

Keywords: Fixed Consumable Electrode Filling Method, ESC, Filling Channel, Numerical Simulation

\section{固定自耗电极充填法熔铸过程中充填通道的研究}

\author{
王珺 ${ }^{1}$, 常国威 ${ }^{1}$, 陈瑞 ${ }^{2}$ \\ ${ }^{1}$ 材料科学与工程学院, 辽宁工业大学, 锦州, 中国 \\ 2沈阳铸造研究所, 沈阳, 中国
}

邮箱

cailiaowangjun@sina.com(王珺), cgw@1nut.edu.cn(常国威), chenrui320@sina.com(陈瑞)

\begin{abstract}
摘要: 本研究以自耗电极、渣池和铸锭为研究对象, 根据渣池对流传热方程、铸锭的导热方程和熔体流动方程建立数 学模型, 施加恰当的电磁场和热场边界条件, 利用大型有限元分析软件ANSYS研究固定自耗电极充填法电渣熔铸过程中 工艺参数对充填通道的影响。研究表明, 对于结晶器内腔尺寸为 $400 \times 70 \times 400$ 的板形铸锭, 当移动自耗电极电流密度 大于等于 $0.5 \mathrm{~A} / \mathrm{mm}^{2}$ 、电极间距小于等于 $0.15 \mathrm{~m}$ 且电极厚度大于等于 $0.035 \mathrm{~m}$ 时, 两金属熔池连通, 移动自耗电极熔化的金 属熔体通过充填通道顺利地补充到固定自耗电极下金属熔池内，确保铸件在稳定的电渣条件下凝固成型。充填通道的 最小截面积随着移动自耗电极电流密度和自耗电极的横截面积的增大而增大。
\end{abstract}

关键词：固定自耗电极充填法，电渣熔铸，充填通道，数值模拟 


\section{1. 引言}

通过电渣熔铸冶炼方法获得的铸件有害元素含量低、 铸造缺陷少、没有成分偏析现象, 并且生产设备简单、实 际操作方便、工艺稳定性高、在高品质铸件的生产方面有 着不可替代的优势, 因此受到人们的重视[1-6], 随着航空 航天、水利发电、大型船舶制造业的迅速发展, 对电渣熔 铸产品的需求不断增大。

常规的电渣熔铸过程中, 自耗电极被公共型腔中的液 态渣加热熔化, 形成的金属熔滴穿过渣池进入结晶器; 当 铸件的形状比较复杂, 由于公共型腔过小或无公共型腔, 普通的电渣熔铸方法根本无法生产示。这些原因使得只有 形状简单的铸件才能采用电渣熔铸冶炼方法, 熔铸形状复 杂的铸件问题一直是电渣学者们的一块心病。

针对形状复杂铸件的电渣熔铸问题, 本课题组提出了固 定自耗电极充填法熔铸新工艺, 其原理如图1所示。该方法 中固定自耗电极本身无法充填整个铸件结晶器的型腔, 缺失 的部分由移动自耗电极熔化的熔体通过充填通道进行补充, 因此充填通道直接影响熔铸过程的稳定性。为了确保移动自 耗电极熔化的金属熔体顺利地对固定自耗电极下金属熔池 进行充填, 必须研究工艺参数对充填通道的影响。研究成果 可为形状复杂铸件工艺辅助结构的设计和优化熔铸工艺、确 定补偿固定自耗电极充填法电渣熔铸复杂形状铸件的自动 控制方法提供理论依据, 对电渣冶金的发展具有推动作用, 所以研究具有重要的科学意义与实际应用价值。

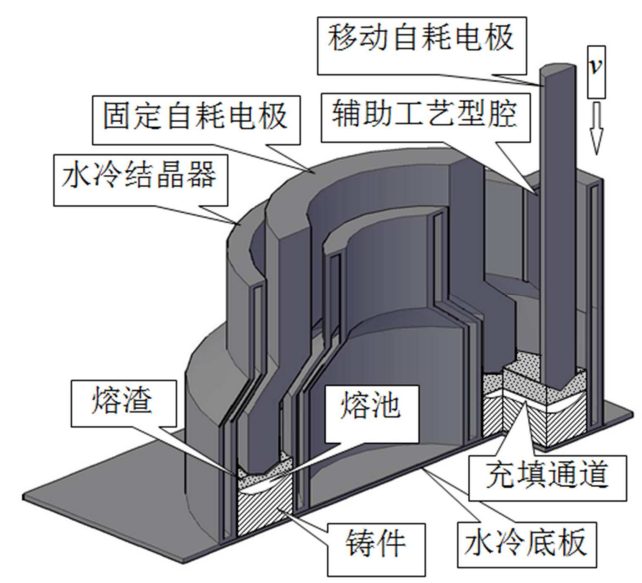

图1 复杂形状铸件固定自耗电极充填法电渣熔铸过程示意图。

20 世纪中叶, 人类工业进行了第三次革命, 电子计算 机技术飞速发展, 人们研发出许多模拟仿真软件 (CAE), 电渣冶金学者利用这些模拟仿真软件对金属熔池的形状 进行了大量的研究, 研究成果有了突破性的进展。

陈元元 [7]以热传导方程为基础建立了电渣重熔的数 学模型并用Visual Basic语言自编了计算程序模拟了凝固 过程中熔池的形状和铸锭的温度分布。马新生 [8]采用数值 模拟的方法对不同渣池深度条件下渣池温度场的变化情 况进行了研究, 发现随着渣池深度的增加, 金属熔池变浅。 钱风娟 [9]利用有限元模拟软件模拟电渣熔铸过程, 得到金 属熔池深度随渣池深度的变化规律。张华 [10]模拟了水轮 机导叶的熔铸过程, 模拟结果与实验结果基本吻合。刘双
[11]结合动量和能量方程, 研究了渣池深度和侵入深度对 金属熔池形状的影响。李宝宽 [12]通过界面移动法和元胞 自动机与有限元相耦合的方法模拟了低碳马氏体不锈钢 ZG06Cr13Ni4Mo电渣熔铸稳定阶段金属熔池形状, 与实验 结果对比发现两者基本吻合。杨明 [13]利用数值模拟与实 验相结合的方法研究了固定自耗电极充填法熔铸初期铸 锭底部形状。对比模拟结果与实验结果, 发现两者基本一 致。

以前人的研究为基础, 本文作者用数值模拟与实验相 结合的方法对固定自耗电极充填法电渣熔铸过程中金属 熔池形状进行了研究, 结果表明, 模拟结果与实验结果基 本吻合, 如图2所示。固本文直接采用已有的数值模拟方 法研究工艺参数对固定自耗电极充填法电渣熔铸过程充 填通道的影响。

\section{2. 数值模拟}

\section{1. 基本假设}

按照抓住主要因素、忽略次要因素的原则, 对模型进 行如下合理的基本假设:

（1）在工艺开始时, 以一个特征初始温度代替不均 匀的温度场;

（2）在工艺进行过程中，忽略金属熔滴在渣中的运 动和对熔铸系统的影响;

(3) 自耗电极在一个较短时间段内与结晶器保持相 对静止, 反应过程为准稳态过程;

（4）电极顶部绝热, 温度为室温且保持不变;

(5) 钢和熔渣的物性参数只和温度有关, 且各部分 有关物性参数可设为常数, 具有均匀性和各向同性;

（6）金属熔池与渣池中的对流换热简化为传导传热 和对流换热系数以有效导热系数来代替。

\section{2. 数学模型}

(1) 熔池与铸锭内导热方程 稳态时熔池与铸锭内热量控制方程为

$$
\rho_{i} C_{p i} v_{z} \frac{\partial T}{\partial z}=\lambda_{i}\left(\frac{\partial^{2} T}{\partial x^{2}}+\frac{\partial^{2} T}{\partial y^{2}}+\frac{\partial^{2} T}{\partial z^{2}}\right)+q_{i}
$$

式中, $\rho$ 为密度, $\mathrm{kg} \cdot \mathrm{m}^{-3} ; C_{P}$ 为恒压热容, $\mathrm{J} . \mathrm{kg}^{-1} \cdot \mathrm{k}^{-1}$; $v_{z}$ 为铸锭的上涨的速度, $\mathrm{m} . \mathrm{s}^{-1} ; T$ 为温度, ${ }^{\circ} \mathrm{C} ; \lambda_{i}$ 为导热系 数, $\mathrm{w} \cdot \mathrm{m}^{-1} \cdot \mathrm{k}^{-1} ; x 、 y 、 z$ 为直角坐标, $\mathrm{m} ; q_{i}$ 为初生的焦耳 热, $\mathrm{J} . \mathrm{m}^{-3} \cdot \mathrm{s}^{-1} ; i=1, \mathrm{~m}, \mathrm{~s}$, 其中 $1, \mathrm{~m}, \mathrm{~s}$ 分别表示金属熔池部分、 为固液两相区、铸锭。

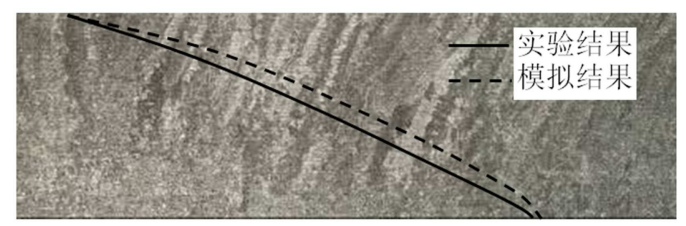

图2 固定自耗电极充填法电渣熔铸熔池形状模拟与实验结果。 
（2）渣池内对流传热控制方程

(1)传热方程

渣池的传热由对流传热方程来确定，即

$$
\rho C_{p}(v \nabla T)=\nabla K_{e f f} \nabla T+q_{v}
$$

式中, $\rho$ 为熔的密度, $\mathrm{kg} . \mathrm{m}^{-3} ; C_{P}$ 为熔渣的恒压热容, $\mathrm{J} . \mathrm{kg}^{-1} \cdot \mathrm{k}^{-1} ; v$ 为熔渣的流动速度, $\mathrm{m} . \mathrm{s}^{-1} ; K_{\text {eff }}$ 为熔渣的导热系 数, $\mathrm{w} \cdot \mathrm{m}^{-1} \cdot \mathrm{k}^{-1} ; q_{v}$ 为熔渣产生的焦耳热, $\mathrm{J} . \mathrm{m}^{-3} \cdot \mathrm{s}^{-1}$ 。

(2)熔渣流动速度方程

熔渣的流动速度 $\mathrm{v}$ 由下列方程确定, 即

$$
\rho(v \nabla) v=-\nabla P+\nabla\left(\mu_{e f f} \nabla v\right)+F
$$

式中, $P$ 为压力, $P a ; \mu_{e f f}$ 为熔渣粘度, Pa.s; $F$ 为体积 力。

(3)体积力方程

$$
F=J \times B+\rho g\left[1-\beta\left(T-T_{0}\right)\right]
$$

式中, $J$ 为电流密度, $\mathrm{A} \cdot \mathrm{m}^{-2} ; \mathrm{B}$ 为磁感应强度, $\mathrm{T} 。 J$ 与 $B$ 分别由法拉第电磁感应定律和欧姆定律确定。

\section{3. 实体模型与网格划分}

由于固定自耗电极充填法熔铸新工艺是首次提出的, 为了便于研究, 将复杂铸件简化为板状铸件, 如图3所示。

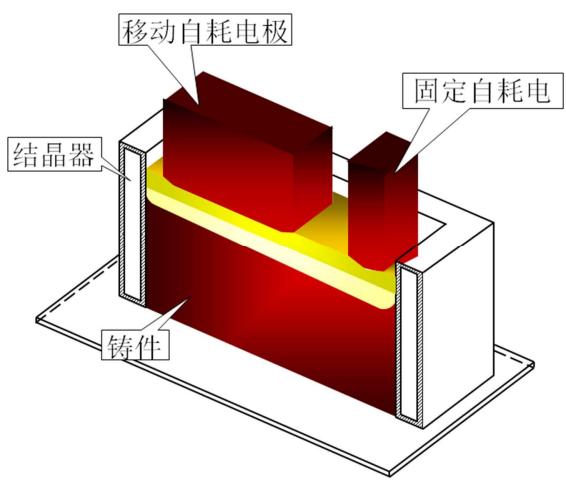

图3 简化后的实体模型。

选取自耗电极、渣池和铸锭部分建立几何模型, 考虑 地球的磁场影响, 在模型外围取 5 倍于熔铸系统的空气层, 熔铸系统实体模型与网格划分如图4所示。

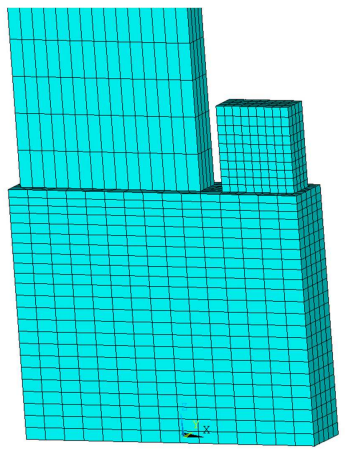

图4 实体模型与网格划分。
充填通道最小截面积可以直接反应出单位时间内移 动自耗电极向固定自耗电极填充金属熔体的能力, 充填通 道最小截面积 $\mathrm{A}$ 的示意图以及模型的几何参数如图 5 所示。

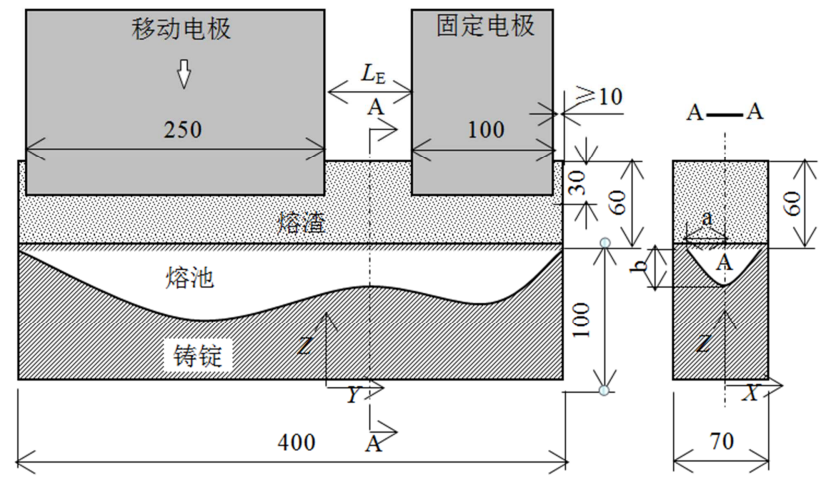

图5 模拟对象的几何参数。

\section{4. 求解过程}

（1）求解方法

本工作选用磁矢量位方法（选用solid97单元）求解电 磁场和焦耳热场, 首先由矢量磁位计算出磁感应强度和电 流密度, 然后将电流密度或电场结果带入焦耳定律公式, 通计算求解得到热功率密度（即焦耳热场分布），通过耦 合计算, 转换单元(使用 solid90), 求解出温度场分布。其 中电极、钢锭和渣层选择时间积分电势 (VOLT)作为自由 度。

（2）边界条件

磁场计算边界条件: 移动电极和固定电极上端面施加 电流, 并分别耦合volt自由度。铸锭底部端面取电位为 0 , 并在空气外表面处设置磁平行边界条件。

温度场的计算主要是考虑电极、电渣和铸锭的边界条 件。电极区域: 自耗电极顶部按实验中实测的温度作为该 边界条件; 自耗电极侧壁按自然对流传热进行处理。渣池 区域: 渣池顶部存在辐射换热和对流换热两种传热方式, 由于自然对流换热量远远小于辐射换热量, 假设渣池上表 面仅发生辐射换热。

设定模拟过程中环境温度为 $20^{\circ} \mathrm{C}$, 冷却水进出口温度 分别为 $20^{\circ} \mathrm{C}$ 和 $40^{\circ} \mathrm{C}$, 冷却水流量为 25 升 $/ \mathrm{min}$, 工艺参数的 调整如表1所示。

表1 工艺参数。

\begin{tabular}{llll}
\hline 序号 & 电流密度 $J\left(\mathrm{~A} / \mathrm{mm}^{2}\right)$ & 电极间距 $L_{\mathrm{E}}(\mathrm{m})$ & 电极厚度 $h(\mathrm{~m})$ \\
\hline 1 & 0.4 & 0.06 & 0.03 \\
2 & 0.5 & 0.09 & 0.035 \\
3 & 0.6 & 0.12 & 0.04 \\
4 & 0.7 & 0.15 & 0.045 \\
5 & 0.8 & 0.18 & 0.05 \\
\hline
\end{tabular}

（3）物性参数

渣和钢锭的物性参数如表 2 所示。 
表2 物性参数。

\begin{tabular}{lll}
\hline 物理性质 & 渣 $[14]$ & 钢 \\
\hline 电导率 $/ \Omega^{-1} \mathrm{~m}^{-1}$ & $3.22 \times 10^{5}$ & $7.14 \times 10^{5}$ \\
磁导率 $/ \mathrm{Hm}-1$ & $1.257 \times 10^{-6}$ & $1.257 \times 10^{-6}$ \\
热传导系数 $/ \mathrm{Wm}^{-1} \mathrm{~K}^{-1}$ & 10.46 & 35.1 \\
比热 $/ \mathrm{Jkg}^{-1} \mathrm{~K}^{-1}$ & 1255 & 828 \\
密度 $/ \mathrm{kgm}^{-1}$ & 2800 & 7800 \\
熔点 $/ \mathrm{K}$ & 1673 & 1773 \\
\hline
\end{tabular}

(4) 模拟流程

具体模拟过程如图6所示。

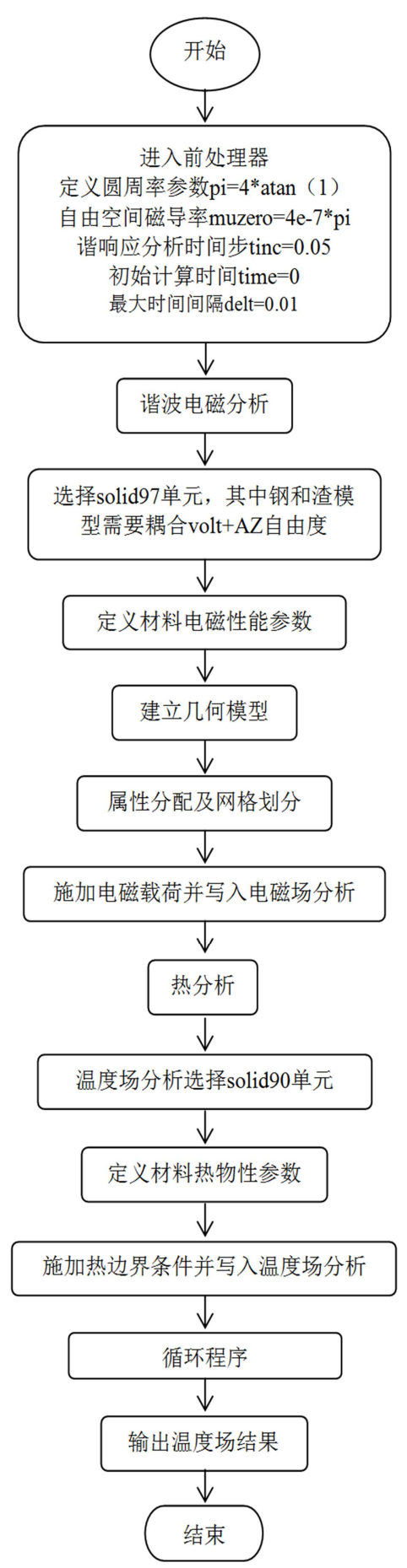

图6 模拟流程。

\section{3. 结果与讨论}

\section{1. 电流密度的影响}

电极厚度为 $0.03 \mathrm{~m}$ 、两电极间距为 $0.06 \mathrm{~m}$ 时, 移动自耗 电极电流密度对充填通道的最小截面积影响如图7所示。 随着移动自耗电极电流密度的增大, 充填通道的最小截面 积增加。当移动自耗电极电流密度增大到 $0.7 \mathrm{~A} / \mathrm{mm}^{2}$ 时, 充 填通道的最小截面的增大主要依靠充填通道深度的增加。

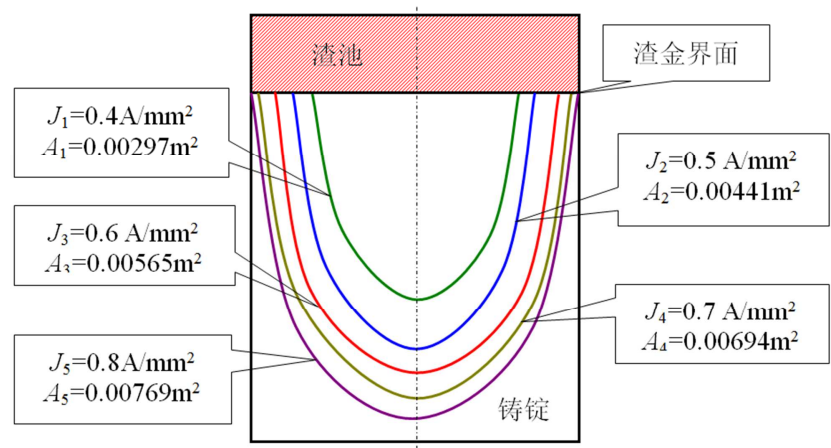

图7 移动自耗电极电流密度对充填通道最小截面积的影响。

不同移动自耗电极电流密度下填充通道最小横截面 积的变化曲线如图8所示。从图中可以看出, 随着移动自 耗电极电流密度的增大, 填充通道最小横截面积不断增大。

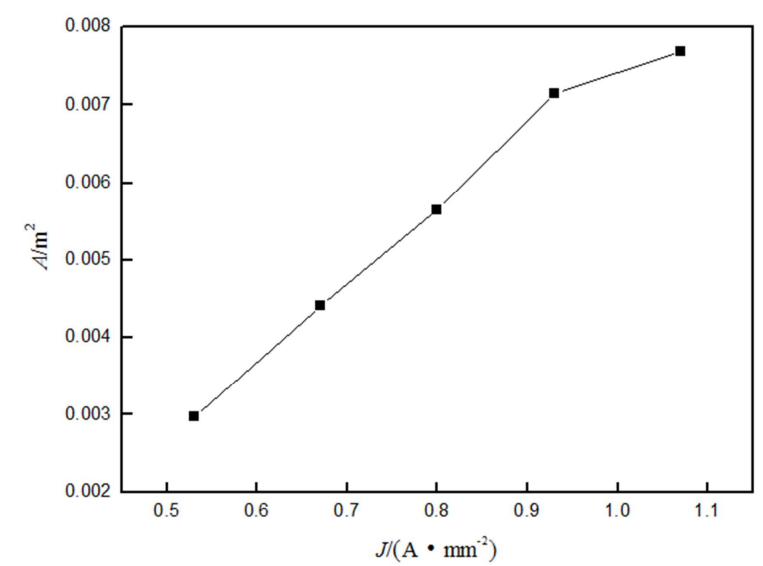

图8 不同移动自耗电极电流密度下填充通道最小横截面积的变化曲线。

综上所述, 填充通道的最小横截面积随着移动自耗电 极电流密度的增大而增大, 单位时间内移动自耗电极向固 定自耗电极填充金属熔体的能力增强。

\section{2. 电极间距的影响}

为了便于研究电极间距对填充通道的影响, 应尽量减 小其他工艺参数对填充通道的影响, 故选取电极厚度的中 间值 $0.04 \mathrm{~m}$ 。移动自耗电极电流密度分别为 $0.4 \mathrm{~A} / \mathrm{mm}^{2}$ 、 $0.5 \mathrm{~A} / \mathrm{mm}^{2} 、 0.6 \mathrm{~A} / \mathrm{mm}^{2} 、 0.7 \mathrm{~A} / \mathrm{mm}^{2}$ 和 $0.8 \mathrm{~A} / \mathrm{mm}^{2}$ 时, 不同电 极间距下填充通道最小横截面积的变化曲线如图9所示。

当移动自耗电极电流密度小于等于 $0.5 \mathrm{~A} / \mathrm{mm}^{2}$ 时且电 极间距超过 $0.15 \mathrm{~m}$ 时, 充填通道最小截面积几乎等于 0 , 说 
明此时移动自耗电极无法向固定自耗电极充填金属熔体, 这种工艺参数下固定自耗电极充填法电渣熔铸失去意义。

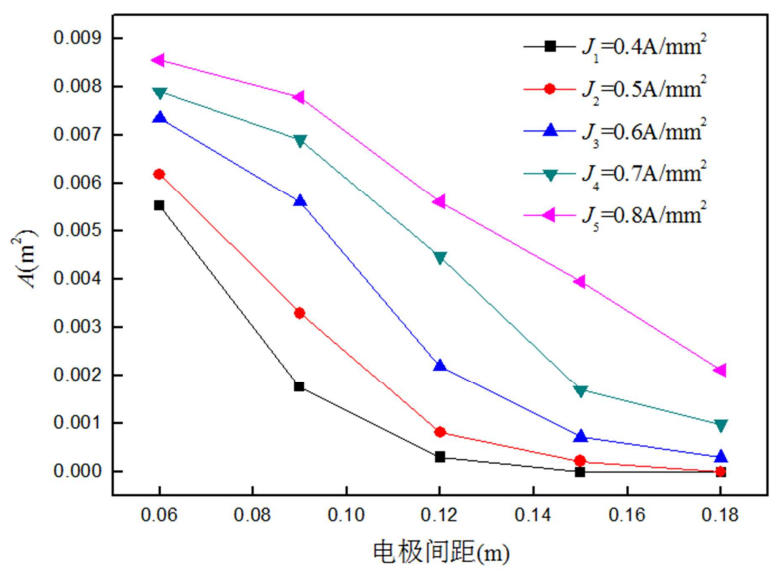

图9 电极间距对充填通道最小截面积的影响。

\section{3. 电极厚度的影响}

通过分析电极间距对填充通道的影响, 发现移动自耗 电极和固定自耗电极之间的最大距离为 $0.15 \mathrm{~m}$, 故选取电 极间距为 $0.15 \mathrm{~m}$ 。当移动自耗电极电流密度分别为 $0.4 \mathrm{~A} / \mathrm{mm}^{2} 、 0.5 \mathrm{~A} / \mathrm{mm}^{2} 、 0.6 \mathrm{~A} / \mathrm{mm}^{2} 、 0.7 \mathrm{~A} / \mathrm{mm}^{2}$ 和 $0.8 \mathrm{~A} / \mathrm{mm}^{2}$ 时, 不同电极厚度下填充通道最小横截面积的变化曲线如 图 10 所示。当移动自耗电极电流密度小于等于 $0.5 \mathrm{~A} / \mathrm{mm}^{2}$ 时, 电极厚度不宜小于 $0.035 \mathrm{~m}$ 。

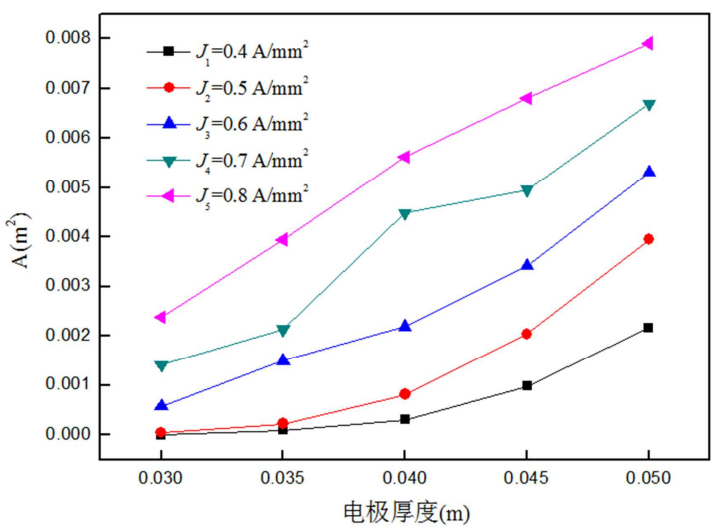

图10 电极厚度对充填通道最小截面积的影响。

从图1可知, 由于铸件形状的变化将导致固定自耗电 极与移动自耗电极间距发生变化, 为了确保移动自耗电极 熔化的金属熔体顺利地补充到固定自耗电极下金属熔池 内, 并保持稳定的电渣熔铸过程, 上述数值计算结果可作 为辅助工艺型腔设计的依据。

\section{4. 结论}

（1）固定自耗电极充填法电渣熔铸时, 随着移动自 耗电极电流密度与充填比的增大、电极间距的减小, 充填 通道最小截面积呈变大的变化规律。
（2）为了确保两金属熔池之间相互连通，固定自耗 电极充填法电渣熔铸 $400 \times 70 \times 400$ 板形铸锭时, 移动自耗电 极电流密度、电极间距以及充填比的最小值分别为: $0.5 \mathrm{~A} / \mathrm{mm}^{2} 、 0.15 \mathrm{~m}$ 和 $0.035 \mathrm{~m}$ 。

\section{致谢}

本文为国家自然科学基金面上项目《固定自耗电极充 填法熔铸复杂形状铸件的基础研究》(51475313)的阶段性 成果之一。

\section{参考文献}

[1] Hoyle G.Electroslag Processes Principles and Practice[M]. London:Aplied Science Publishers, 1983: 12-15

[2] Li Z B.Electroslag Metallurgy Theory and Practice[M]. Beijing:Metallurgical Industry Press, 1996: 25-33

[3] 李正邦.电渣冶金的理论与实践 $[\mathrm{M}]$. 北京:治金工业出版社, 2011: 10-14

[4] 李正邦.电渣治金的发展历程、现状及趋势[J].材料与冶金学 报, 2011,10(S1): 1-7

[5] 李正邦.特种冶金新进展 [J].中国冶金, 2002,57(2)：24-25

[6] 李正邦.21世纪电渣治金的新进展[J].特殊钢, 2004,25(5): $1-5$

[7] 陈元元, 刘喜海, 李宝宽. 电渣重熔钢锭凝固过程数学模拟 软件 [J].钢铁研究学报, 2005, 17(6): 30-33

[8] 马新生, 耿茂鹏, 尧军平, 饶䂞, 杨小军. 电渣熔铸中渣池 深度对渣池温度场影响的模拟研究 [J]. 铸造技术, 2005, 26(12): 1112-1115

[9] 钱风娟, 耿茂鹏, 饶否. 电渣熔铸中渣池深度对金属熔池 深度影响的有限元模拟 [J]. 南昌大学学报, 2006, 20(6): 591-596

[10] 张华, 关洋, 陈瑞, 孙逊, 宋照伟.电渣熔铸导叶数值模拟 技术研究 [J].铸造, 2013,62(7): 637-640

[11] 刘双, 贺铸, 蔡辉, 王强, 李宝宽.不同工艺参数下电渣重 熔过程的数值模拟 [J].钢铁研究学报, 2015, 27(10): 26-33

[12] Liu F B, Chen X, Jiang Z H, Li H B, Deng X.Numerical simulation of solidification structure during electro slag remelting casting of ZG06Cr13Ni4Mo ingot based on CAFE and moving boundary method[J].Ironmaking and Steelmaking, 2016, 5(16): 53-62

[13] 杨明. 固定自耗电极充填法熔铸的数值模拟 [D]. 锦州: 辽宁 工业大学, 2017: 31-49

[14] Qiang WANG, Baokuan LI. Numerical Investigation on the Effect of Slag Thickness on Metal Pool Profile in Electroslag Remelting Process. ISIJ Int., 2016,56: 282-287 